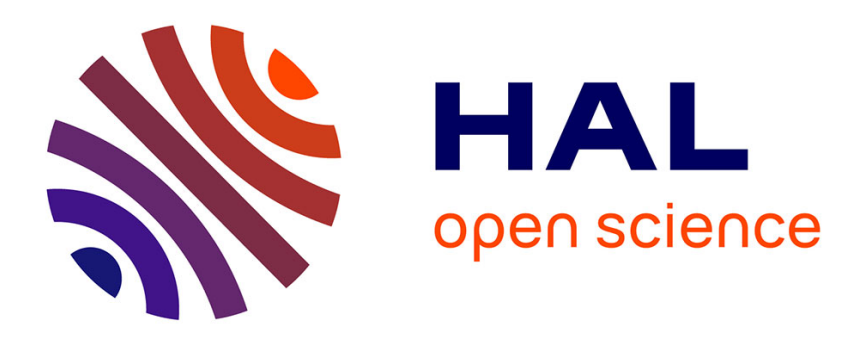

\title{
Social Welfare to Assess the Global Legibility of a Generalized Map
}

Guillaume Touya

\section{To cite this version:}

Guillaume Touya. Social Welfare to Assess the Global Legibility of a Generalized Map. GIScience 2012, Sep 2012, Columbus, Ohio, United States. pp.198-211. hal-02297732

\section{HAL Id: hal-02297732}

\section{https://hal.science/hal-02297732}

Submitted on 27 Sep 2019

HAL is a multi-disciplinary open access archive for the deposit and dissemination of scientific research documents, whether they are published or not. The documents may come from teaching and research institutions in France or abroad, or from public or private research centers.
L'archive ouverte pluridisciplinaire HAL, est destinée au dépôt et à la diffusion de documents scientifiques de niveau recherche, publiés ou non, émanant des établissements d'enseignement et de recherche français ou étrangers, des laboratoires publics ou privés. 


\title{
Social Welfare to Assess the Global Legibility of a Generalized Map
}

\author{
Guillaume Touya \\ Laboratoire COGIT, IGN, 73 avenue de Paris, 94165 Saint-Mandé France \\ Name.surnamedign.fr
}

\begin{abstract}
Cartographic generalization seeks to summarize geographical information from a geo-database to produce a less detailed and readable map. The specifications of a legible map are translated into a set of constraints to guide the generalization process and evaluate it. The global evaluation of the map, or of a part of it, consisting in aggregating all the single constraints satisfactions, is still to tackle for the generalization community. This paper deals with the use of the social welfare theory to handle the aggregation of the single satisfactions on the map level. The social welfare theory deals with the evaluation of the economical global welfare of a society, based on the individual welfare. Different social welfare orderings are adapted to generalization, compared and some are chosen for several generalization use cases. Experiments with topographic maps are carried out to validate the choices.
\end{abstract}

Keywords: map generalization, evaluation, social welfare, constraints

\section{Introduction}

Cartographic generalization is a process that seeks to summarize geographical information from a geo-database in order to produce a less detailed and readable map. Automatic generalization processes were necessary to ease the production of map series and are growingly required nowadays with the development of on-demand mapping. Past research proposed many different approaches to automatically generalize maps [1]. Automatic generalization processes require evaluation procedures both to control and to validate [2]. On the one hand, assessing where the map needs to be generalized is necessary to control which algorithm to use (i.e. enlarge, displace...) and where to use it. On the other hand, an automatic process needs to know if the generalization it performed was successful to validate itself and avoid as much as possible manual post-correction. The automatic evaluation of single specifications in the map, like the minimum area of displayed buildings, has been well tackled [3,4]. But a map is composed of a very large amount of such specifications and it is very difficult to assess the global quality of a generalized map [3]. Such evaluation is now carried out by human cartographers. Is it possible to aggregate the single evaluations into a unique value that says 'this map is perfectly generalized according to the specifications'? Is it possible to compare two map generalization alternatives?

This paper tries to answer to these questions, drawing its inspiration from collective welfare theories in economy. Collective welfare assesses the welfare of a human society from individual welfare [5]. In map generalization evaluation, the specifications for each object can be considered as the individuals in collective welfare.

The second part of the paper deals with the description of the application problem, the global assessment of generalized maps. The third part gives an overview of the collective social welfare theories and describes a benchmark developed to compare social welfare orderings in relation to map specifications satisfaction. The fourth part shows some experiments of our approach inside the automatic generalisation model CollaGen [1]. The last part draws some conclusions and explores further research.

\section{Global Assessment of Generalized Maps}

As research and technologies allow going further and further towards the automatic generation of maps at different scales, the processes require advanced self evaluation tools. Self evaluation is necessary both to control and validate an automatic process [2]. Indeed, it is necessary to know, at some point of an automatic process, what was well generalized and what is left to do; and as processes success is very dependent on the geographical context they are applied on, it is necessary to rely on self evaluation to validate the result of a process. The point is not to evaluate the quality of generalized data [6], but to answer the following questions: Does the generalized map truly reflect the initial data? Are the transformations due to generalization acceptable? Are the map specifications met? The first part of the section reports related work on generalization evaluation. The second part deals with global assessment from a set of constraints. The third part details three use cases. 


\subsection{Related Work}

Ruas and Mackaness [2] give an overview of past research on the evaluation of automatic map generalization. They state that a generalization evaluation process should include three components: a representation of the real world to verify that generalized data reflect the initial information, a representation of the user's needs (i.e. the expected level of detail of the generalized map and the objectives of the map), and a representation of the rules of cartography and database integrity.

A first method is to identify the geographic characters that bear the user's needs and the cartographic rules, and to assess if generalization is successful according to each character, thanks to spatial measures. For instance, it consists in measuring if the buildings are large enough or if the roads coalesce. Similarly, in the AGENT automatic generalization model [7], each object is able to evaluate its characters in relation to the specifications. In order to benchmark existing generalization algorithms, a template for sharing the measures to evaluate the most common characters of geographic objects was proposed [8]. More recent research went further in this approach, defining evolution function for each of the character [3]. Evolution function give the expected final value of a character according to the initial value and thresholds extracted from the specifications. Fig. 1 shows an example of evolution function for the area of buildings. The evolution functions can be determined using cartographers knowledge or by reverse engineering [4]. Experiments show that such an approach allows evaluating individually every element of the user's needs, the cartographic rules and even the respect of the initial data [4]. Nevertheless, difficulties remain in the evaluation of characters of complex group of objects like building alignments [9].

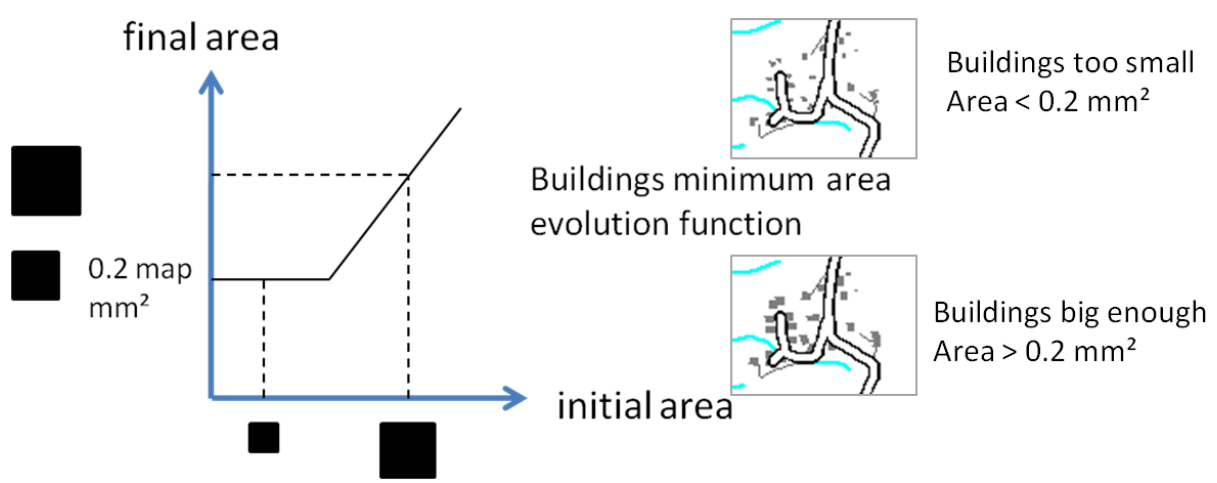

Fig. 1. Evolution function of a constraint on building minimum area.

Although it allows a fine description of the legibility of a generalized map, the previous approach only provides the building blocks for a global evaluation of the map. The aggregation of these elementary evaluations is stated as an important issue: a simple solution is to express the elementary evaluation in unique Likert-like scale [10] and to compute the mean (or median) of the elementary evaluations, weighted by the relative importance of the characters $[1,3,7]$. Without satisfying automation, past research resorted to human cartographers' evaluation, using ranking templates $[8,4]$.

Relying on human intervention is prohibited in automatic processing, so it is necessary to figure out how to globally assess a generalized map. The next section describes the framework built to study the global evaluation and why the simple mean of elementary evaluations is not sufficient.

\subsection{Approach: Global Assessment from a Set of Monitors}

In order to globally assess generalized maps, we propose a framework that benefits from the past research on the elementary evaluation of characters. In this framework, constraints are used to represent the user's needs and the cartography rules. Constraints have proved to be the most relevant way to model the specifications [11] and make consensus in the generalization research community to serve as input of generalization processes [1, 4]. A constraint constrains the value of character of a map object, once generalized. (C1): "Buildings area should be bigger than 0.2 map $\mathrm{mm}^{2}$ " and $(\mathrm{C} 2)$ : "Roads initial general shape should be maintained" are two examples of generalization constraints. To ensure a representation of the real world [2], a geographical objects ontology can be used to express the constraints [1].

In the proposed framework, the constraints that represent user's needs are monitored by constraint monitors like in [1]. Constraints monitors locally convey the evaluation of the constraint satisfaction for a given object. As suggested in [7], constraint monitors satisfaction is comprised in an integer interval, between 1 and 8 . The integer values make comparisons and interpretation easier, compared to real values. The number of possible values was 
five in [7] but is extended here to eight in order to make the expression of small improvements easier. Qualitative descriptions are associated to the satisfaction value as abstraction of measures helps the understanding of the value [12] (e.g. 1 is represented by "Unacceptable" and 8 by "Perfect"): the satisfaction scale is thus Likert-like [10]. In order to compute its satisfaction, each monitor is endowed with its own method that compares current and goal values. For instance, $(\mathrm{C} 1)$ is monitored by as many monitors as buildings in the map (e.g. if there are 500 buildings in the map, 500 monitors monitor $(\mathrm{C} 1)$ ), and the monitor uses the evolution function from Fig. 1 to assess satisfaction: if the current building area is equal to the goal area of the evolution function, satisfaction is "Perfect" (i.e. 8), if the area is bigger than $60 \%$ of goal area, satisfaction is "Fair" (i.e. 4), etc.

In this framework, the global assessment of generalized maps comes to evaluate the distribution of monitors' satisfaction. The utilitarian method (i.e. using the satisfactions mean [13]) as proposed by [1, 3, 7] is not enough to assess globally a generalized map. Fig. 2 helps to illustrate this statement: two generalization alternatives are evaluated with nine monitors that monitor constraints on proximity between buildings and proximity between building and road symbols. If the mean of satisfaction is used, the $\mathrm{n}^{\circ} 2$ is preferred (7.2 against 5.8) whereas a human cartographer would prefer $n^{\circ} 1$, especially for a direct printing of the map, as no big legibility problem remain.

The constraints do not all have the same importance in a generalized map [4,7,11] (e.g. minimal size is more important than preserving initial position), so the satisfaction distributions are weighted by constraint importance. But for the sake of simplicity, in this paper, all constraints have equal importance.

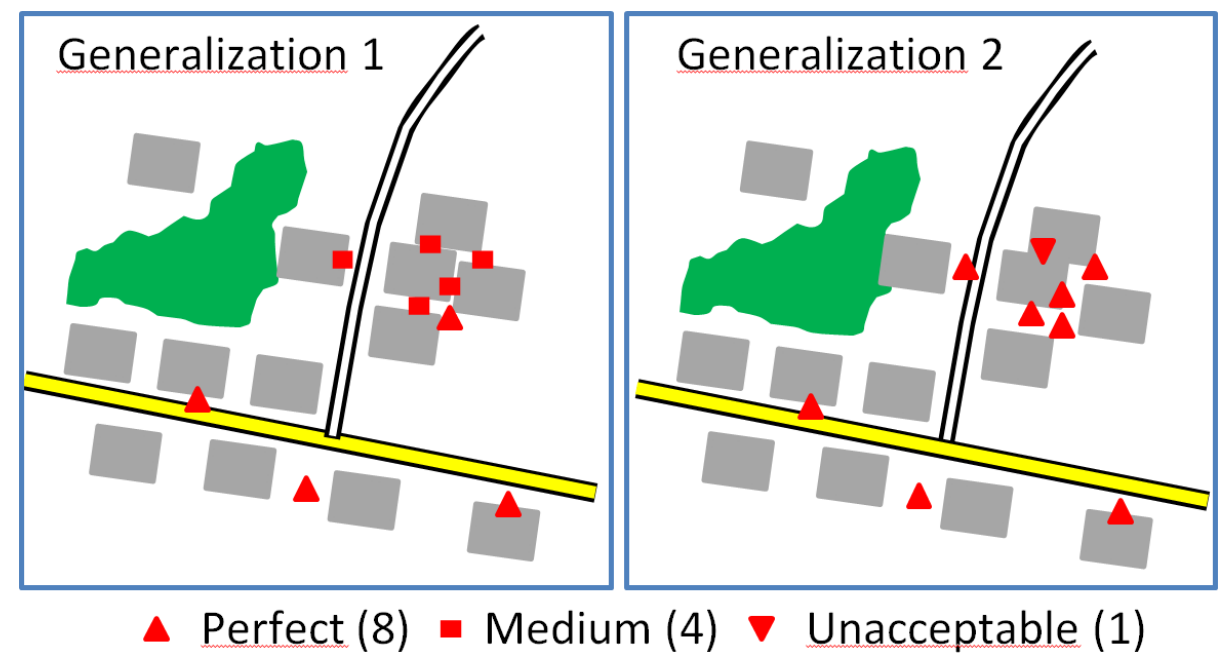

Fig. 2. Two generalization solutions evaluated by a sample of the constraint monitors.

\subsection{Use Cases to Distinguish}

The global assessment of a generalized map (or of a generalized part of a map) can be necessary in several situations, when generalization is carried out by automatic processes. Thus, three use cases, where global assessment is required, have been identified: evaluating a final output (ready to print), evaluating a final output with possible manual post-editing and comparing the legibility of the map with the previous step in an iterative automatic process.

The first use case is the evaluation of a final output, a map ready to be printed without further correction of remaining legibility problems. In this use case, major legibility problems that blur map interpretation should be avoided. Considering distributions, it means that constraint satisfaction distributions with the more perfect satisfaction are preferred as the perfect satisfaction is the only one that guarantees no legibility inconvenience. Moreover, distributions with less unacceptable satisfactions are preferred as such satisfactions are supposed to represent very inconvenient legibility problems while the medium satisfactions represent minor problems that do not disturb global legibility much. In this use case, in Fig. 2, (1) would be preferred to (2). 


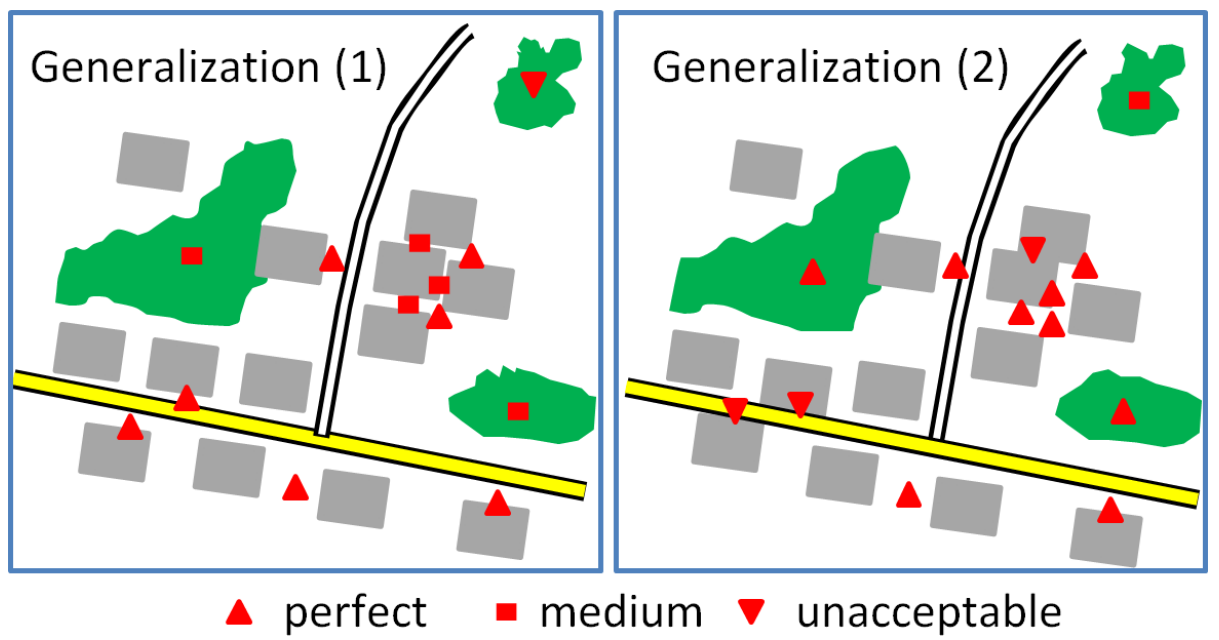

Fig. 3. Two generalizations that illustrate the second use case: manual editing. $\mathrm{N}^{\circ} 2$ is preferred to $\mathrm{n}^{\circ} 1$ as fewer manual editing are required in this solution.

The second use case is the evaluation of a final output of an automatic process but with possible manual editing of the remaining legibility problems. This use case is very important as most of actual map production lines based on automatic generalization resort to manual post-editing of the automatically produced map. This case is the opposite of the previous one as distributions with marked differences in satisfaction values and less medium values are preferred. Indeed, all imperfect satisfaction monitors (even medium ones) require manual edition of the corresponding legibility problem. Moreover, the correction of a legibility problem identified by medium satisfaction is as long as a big legibility problem. Thus, in the example of Fig. $3, n^{\circ} 2$ is preferred as it only requires 4 manual edits ( 3 unacceptable satisfactions and 1 medium one) while 6 are required for $n^{\circ} 1$ ( 1 unacceptable satisfaction but 5 medium ones).

The last use case is the evaluation of a positive evolution of satisfaction compared to the previous state of the map in an iterative automatic process. Contrary to the second use case, the global legibility of the map is not assessed but only the global evolution of satisfactions. In this case, the distributions with less low satisfactions and more high satisfactions are preferred, whatever the evolution on medium satisfaction is.

We believe that the simple mean of the satisfactions is not enough to evaluate globally a generalized map in the three use cases, and that collective welfare theories may provide alternatives. In order to prove the assertion, we carried out a four step reasoning: first implement a library of social welfare orderings (i.e. potential alternatives to mean) from literature; then define a set of toy distributions that illustrate the diversity of constraints distributions; analyze how social welfare alternatives order the toy distributions to choose one adapted to each use case; finally, prove choice validity by testing on real generalized data. The next section introduces the theories of collective welfare, and describes the first three steps of our reasoning.

\section{Theories of Collective Social Welfare}

\subsection{Collective Welfare and Social Welfare Ordering}

Microeconomic analysis and economic theories assume that each individual tries to maximize its own preferences, its welfare, often assimilated to the money earned by the individual. Then, collective welfare is the aggregation of the individual welfare of every member of a society, and economical policies intend to maximize collective welfare [5]. We assume that the global assessment of generalized maps can be considered as a collective welfare problem where the individuals are the constraint monitors and their utility is their satisfaction. Cardinal welfarism studies social welfare orderings (SWOs) in order to analyze the collective welfare of a society. SWOs allow comparing two societies according to collective welfare, using the individual welfare. Different SWOs may convey different perspectives on collective welfare favoring either the total society welfare or the decrease of welfare difference between individuals. For a given individual utilities distribution $\left(\mathrm{u}_{1}, \mathrm{u}_{2} \ldots \mathrm{u}_{\mathrm{n}}\right)$ noted $\left(u_{i}\right)$ and a second distribution $\left(u_{i}^{\prime}\right)$, a social welfare ordering provides an order relation, i.e. allows to assess that $\left(u_{i}\right)$ is preferred to $\left(u_{i}^{\prime}\right)$, which can be noted:

$$
\left(u_{i}\right)>\left(u_{i}^{\prime}\right)
$$


A great diversity of social welfare orderings can be defined to compare distributions of individual utility. For instance, the utilitarian social welfare ordering, coming from Bentham philosophy [13], considers the sum of individual utilities to compare collective distributions (Equation 2).

$$
\left(u_{i}\right)>\left(u_{i}^{\prime}\right) \Leftrightarrow \sum \mathrm{u}_{\mathrm{i}}>\sum \mathrm{u}_{\mathrm{i}}^{\prime}
$$

A Collective Utility Function (CUF) may be related to a social welfare ordering. The CUF gives a real value representing the value of the collective welfare according to the scale of utilities. For instance, the collective utility function related to the utilitarian social welfare ordering of Equation 2 is the mean of the individual utilities. SWOs and their CUF are not exclusively used in economy but also in computer science and particularly multi-agents systems research in order to solve resource allocation problems that require fair division of resources [14].

Historically, there are three approaches to collective welfare: the utilitarian, the egalitarian and the Nash orderings. The utilitarian orderings introduced above, consider the sum of individual utilities as the basis for SWOs and related CUF. Such an approach will prefer a society with equally distributed unsatisfied individuals and very satisfied individuals, compared to one where all the individual utilities are equal to the mean. Egalitarism orderings follow the justice principles of Rawls [15]. In order to favor fair distributions, egalitarian orderings will compare the least satisfied individuals [5]. Finally, Nash orderings try to balance utilitarian and egalitarian approaches by using the product of individual utilities as the basis for ordering. Nash orderings penalize the distributions with some very unsatisfied individuals.

We developed a library of social welfare orderings, following the three approaches, which convey different behaviours to compare distributions. Within this quite large library, choices can be made to use the most appropriate ordering in order to compare global generalisation results in the proposed use cases. These social welfare orderings and their CUF are described in the next section.

\subsection{A Library of Social Welfare Orderings}

The social welfare orderings described in this section are illustrated with a simple example composed of three distributions of individual utilities, $\mathrm{u}, \mathrm{v}$ and $\mathrm{w}$ (Equation 3). There are five individuals and their utility varies from 1 to 8 .

$$
u=\{1,2,8,8,8\}, v=\{2,3,6,6,6\}, w=\{4,4,4,4,4\}
$$

Utilitarian SWOs. The utilitarian SWOs derive from the classical utilitarian SWO [13] presented in Equation 2: utilities are summed. The utilitarian SWO ranks the $u$ distribution as the best, as the utilities sum is the highest (27 against 23 for $v$ and 20 for $w$ ). A simple alternative to the utilitarian SWO is a powered utilitarian SWO (Equation 4) that penalizes the low utilities when the power parameter is high.

$$
\left(u_{i}\right)>\left(u_{i}^{\prime}\right) \Leftrightarrow\left(\sum \mathrm{u}_{\mathrm{i}}^{\mathrm{p}}\right)^{1 / \mathrm{p}}>\left(\sum \mathrm{u}_{\mathrm{i}}^{\mathrm{p}}\right)^{1 / \mathrm{p}}
$$

The Iso-Elastic SWO is a more egalitarian version of utilitarism when its parameter $p$ tends to infinity (Equation 5). For instance, with 5 as a parameter value, the examples are ranked differently: $w>v>u$.

$$
\left(u_{i}\right)>\left(u_{i}^{\prime}\right) \Leftrightarrow\left(\frac{1}{1-\mathrm{a}} \sum \mathrm{u}_{\mathrm{i}}^{1-\mathrm{a}}\right)>\left(\frac{1}{1-\mathrm{a}} \sum \mathrm{u}_{\mathrm{i}}^{\prime 1-\mathrm{a}}\right) \text { where } \mathrm{a} \in[0,1[\mathrm{U}] 1,+\infty]
$$

The Owa SWOs are a family of orderings that weight each individual utility differently [16]. Depending on the weights assigned to low, mean or high individual utilities, an Owa SWO may derive from utilitarian SWOs and favour some specific distributions (Equation 6).

$$
\text { for a function } \mathrm{w}\left(u_{i}\right),\left(u_{i}\right)>\left(u_{i}^{\prime}\right) \Leftrightarrow \sum \mathrm{w}\left(\mathrm{u}_{\mathrm{i}}\right) \cdot \mathrm{u}_{\mathrm{i}}>\sum \mathrm{w}\left(\mathrm{u}_{\mathrm{i}}^{\prime}\right) \cdot \mathrm{u}_{\mathrm{i}}^{\prime}
$$

Egalitarian SWOs. The egalitarian SWOs follow Rawls principles of justice [15], penalizing the distributions with a low minimum. Most egalitarian SWOs rely on the Leximin order [17]. Equation 7 represents the Leximin order: the $\left(u_{i}\right)$ distribution sorted by ascending order is noted $\left(u_{i}\right)^{*}$. The equation means that distributions are compared by their number of low utilities. With this SWO, the rank of the example distributions is different than the utilitarian ones as $w>v>u$ (Fig. 4).

$$
\left(u_{i}\right)>\left(u_{i}^{\prime}\right) \Leftrightarrow \exists \mathrm{i} \in \llbracket 1, \mathrm{n}-1 \rrbracket /\left(\forall \mathrm{j} \in \llbracket 1, \mathrm{i} \rrbracket, \mathrm{u}_{\mathrm{j}}^{*}=\mathrm{u}_{\mathrm{j}}^{\prime *}\right) \wedge \mathrm{u}_{\mathrm{i}+1}^{*}>\mathrm{u}_{\mathrm{i}+1}^{\prime *}
$$


The Leximin SWO is very strict and alternatives have been developed to weaken its effects while keeping an egalitarian ordering [18]. For instance, a poverty line can be introduced: for a poverty line at utility 2 , the vectors $u$ and $v$ are ordered using the Leximin principle because the lowest different values are below the threshold, while vectors like $w$ are compared on an utilitarian basis as all values are over the poverty line. The poverty line may convey the idea that only the very low individual utilities are unacceptable, which can be useful for our generalization evaluation purpose.

It is worth noting that Leximin-based SWOs, do not have a related CUF, able to help differentiate distributions. As a consequence, the egalitarian SWOs will not be useful in use cases where a CUF is needed (use case 1 and 2).
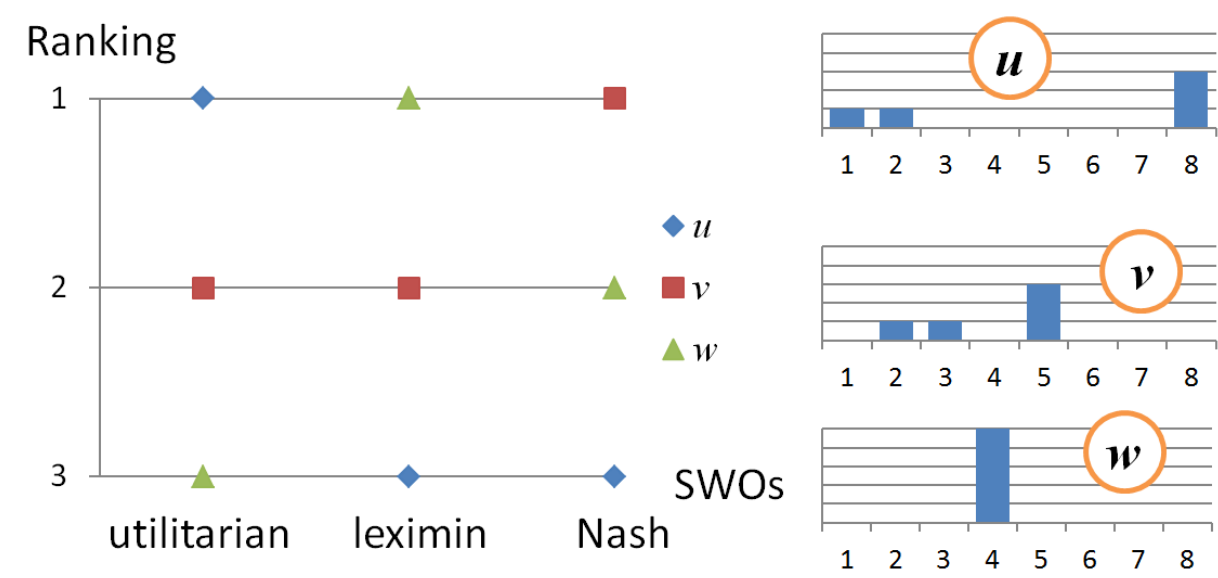

Fig. 4. Summary of the examples distribution orders for the three main SWOs.

Nash SWOs. Finally, the Nash SWOs multiply all individual utilities (Equation 8). Here, the rank of the example distributions is different as $v>w>u$ (Fig. 4).

$$
\left(u_{i}\right)>\left(u_{i}^{\prime}\right) \Leftrightarrow \prod u_{\mathrm{i}}>\prod \mathrm{u}_{\mathrm{i}}^{\prime}
$$

The Nash welfare can be derived to a powered Nash welfare, to favor big utilities (Equation 9). With the powered Nash welfare, the gap between $v$ and $w$ increases, while the gap between $w$ and $u$ decreases.

$$
\left(u_{i}\right)>\left(u_{i}^{\prime}\right) \Leftrightarrow\left(\prod u_{i}^{\mathrm{p}}\right)^{1 / \mathrm{p}}>\left(\prod \mathrm{u}_{\mathrm{i}}^{\prime}\right)^{1 / \mathrm{p}}
$$

\subsection{Social Welfare Ordering Benchmarking}

In order to select the SWOs that could be useful to improve generalization global evaluation in several use cases, it is necessary to comprehend how the SWOs presented in the previous section behave to sort standard distributions. If it is possible to know that a particular SWO favors more than the others a given distribution, this SWO could be used to evaluate generalization in use cases where such a distribution is preferred. This section describes a SWO benchmarking that was carried out to meet this objective, based on toy distributions.

Toy Distributions of Constraint Satisfaction. Toy distributions are distributions of one hundred monitors with specific patterns. The "Medium" toy distribution is composed of 50 monitors with fair satisfaction (4) and 50 with acceptable satisfaction (5), while the "Extreme very good" toy distribution is composed of 30 monitors with satisfaction 1 and 70 with satisfaction 8 (Fig. 5). Considering social welfare, each of the hundred individuals of our toy distribution has a utility that may vary from 1 to 8 . Besides, the 11 toy distributions allow verifying that SWOs do not mix up big trends: the "very good" distributions should always be preferred to medium ones. 


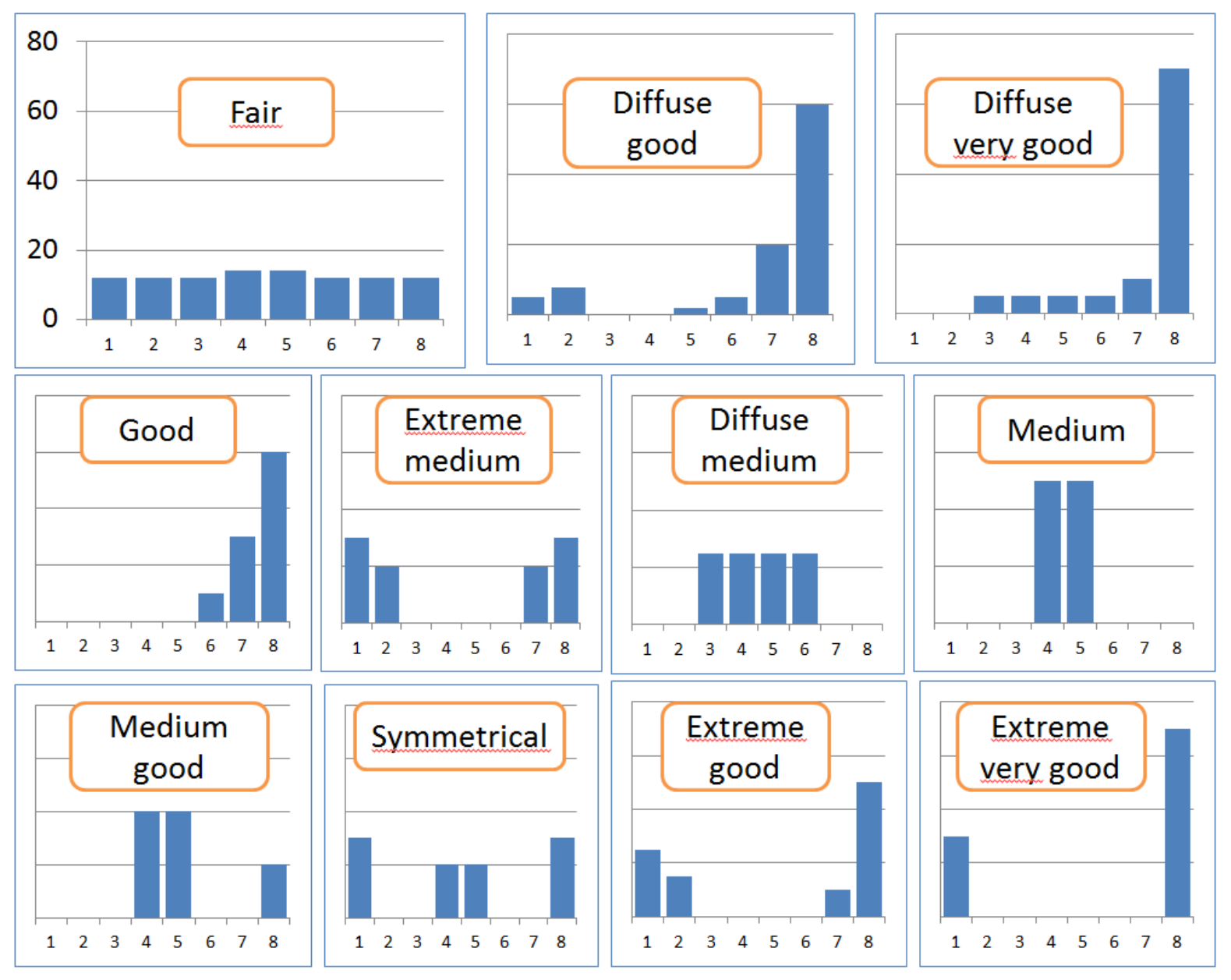

Fig. 5. Utilities histogram (100 individuals) of all the toy distributions.

Ranking the Toy Distributions. Experiments were carried out to benchmark a large set of SWOs with the toy distributions, as the way a SWO ranks the toy distributions illustrates its future behavior with monitor satisfaction distributions. The benchmarked SWOs are thirty ordering methods obtained from the equations previously described, with several parameters for the SWOs that can be parameterized (and also other SWOs from the literature). For each SWO, the result is a list of the toy distributions ordered by preference. For instance, the utilitarian SWO prefers the "Good" toy distribution, then "diffuse very good", "diffuse good", "extreme very good", "extreme good", "medium good", "symmetrical", "medium", "diffuse medium", "extreme medium" and finally the "fair" distribution. Two different analyses were carried out on the ranking lists: a comparison to the mean ordering of each toy distribution and a comparison to the utilitarian list.

The comparison to the mean ordering is computed for each SWO: it is the difference, for each toy distribution (e.g. "fair"), between the mean rank of the distribution in the sorted list of all SWOs ( $9^{\text {th }}$ out of 11 for "fair") and the rank of the toy distribution in the sorted list of a given SWO $\left(11^{\text {th }}\right.$ for "fair" for the utilitarian SWO). This comparison allows finding the favored distributions and the ones that are penalized by a given SWO. For instance, it allows knowing that the powered utilitarian SWO greatly favors more the "medium extreme" distribution (Fig. 5) than the others. 


\begin{tabular}{|c|c|c|c|c|c|c|c|c|c|c|c|}
\hline Evaluation Method & FAIR & $\begin{array}{l}\text { GOOD } \\
\text { LOOSE }\end{array}$ & \begin{tabular}{|l|} 
VERY \\
GOOD \\
LOOSE
\end{tabular} & GOOD & $\begin{array}{l}\text { MEDIUM } \\
\text { EXTREME }\end{array}$ & $\begin{array}{l}\text { MEDIUM } \\
\text { LOOSE }\end{array}$ & MEDIUM & $\begin{array}{l}\text { MEDIUM } \\
\text { GOOD }\end{array}$ & $\begin{array}{l}\text { SYMMET } \\
\text { RICAL }\end{array}$ & $\begin{array}{l}\text { GOOD } \\
\text { EXTREME }\end{array}$ & \begin{tabular}{|l|} 
VERY \\
GOOD \\
EXTREME
\end{tabular} \\
\hline StandardUtilitarianMethod & 0 & 8 & 9 & 10 & 1 & 2 & 3 & 5 & 4 & 6 & 7 \\
\hline PoweredUtilitarianMethod (5.0) & 2 & 0 & 1 & -1 & 4 & -1 & -3 & -2 & 0 & 0 & 0 \\
\hline LeximinPovertyLine (3.0) & 4 & -3 & 0 & 0 & -1 & 4 & 4 & 3 & -3 & -3 & -5 \\
\hline WeakPovertyMean $(2.0,6.0)$ & 4 & -3 & 0 & 0 & -1 & 4 & 4 & 3 & -3 & -4 & -4 \\
\hline OwaWelfare $(4,3,2,1,1,2,3,4)$ & 3 & 0 & 1 & -1 & 4 & -1 & -3 & -3 & 0 & 0 & 0 \\
\hline OwaWelfare $(1,1,1,4,4,1,1,1)$ & 4 & -5 & -3 & -5 & -1 & 6 & 7 & 4 & 3 & -5 & -5 \\
\hline OwaWelfare $(3,3,3,2,2,1,1,1)$ & 4 & -3 & -1 & -3 & -1 & 4 & 6 & 5 & -2 & -5 & -4 \\
\hline IsoElasticMethod $(30.0)$ & 2 & -3 & 0 & 0 & -1 & 4 & 4 & 3 & -3 & -3 & -3 \\
\hline IsoElasticMethod $(0.5)$ & 2 & 0 & 0 & 0 & -1 & 2 & 3 & 2 & -3 & -3 & -2 \\
\hline IsoElasticMethod (0.2) & 2 & 0 & 0 & 0 & -1 & 1 & 1 & 1 & -3 & -1 & 0 \\
\hline NashWelfare & 2 & 0 & 0 & 0 & -1 & 3 & 3 & 2 & -3 & -3 & -3 \\
\hline BernoulliNashWelfare & 4 & 0 & 0 & 0 & -1 & 3 & 3 & 2 & -3 & -4 & -4 \\
\hline AtkinsonWelfare $(0.2)$ & 4 & 0 & 0 & 0 & -1 & 3 & 3 & 2 & -3 & -4 & -4 \\
\hline AtkinsonWelfare $(-10.0)$ & 2 & -3 & 0 & 0 & -1 & 4 & 4 & 3 & -3 & -3 & -3 \\
\hline
\end{tabular}

Table 1. Comparison in toy distribution ranking between standard utilitarian method and a sample of the alternative ones.

The second analysis compares the ranks of every toy distribution in a given SWO preference list to the standard utilitarian preference list ranks (as it the standard method actually used in generalization). The comparison is summarized in Table 1 for a sample of the tested SWOs. This analysis allows finding the distributions that are favored and the penalized ones, by a given SWO compared to the utilitarian SWO. Therefore, the analysis highlights the SWOs that could be used in the use cases where utilitarism fails. It can be noted that the non-utilitarian SWOs can be divided into three groups: the egalitarian, the SWOs balanced between egalitarism and utilitarism (that include Nash, iso-elastic and Atkinson SWOs) and the Owa SWOs that have very unique behaviors depending on their weight parameters. Thanks to both analyses, the next part proposes to match the use cases with the most appropriate SWO.

Relating Social Welfare Orderings to Use Cases. The way the SWOs rank the toy distributions allow to infer their behavior against the four use cases described in section 2. Therefore, it helps us to assign a SWO to use in each use case, in order to improve the current utilitarian methods. The first one is the evaluation of a final output map, ready to be printed on paper or on a screen. According to Table 1, only two SWOs favor the "diffuse very good" distribution over the "good" one: the powered utilitarian SWO and the Owa SWO with high weights on extreme satisfactions. As the Owa SWO favors more the "fair" distribution, it is chosen to assess the final outputs.

The second use case is the evaluation of a map with possibilities of manual post-editing. This use case requires to favor distribution with the fewest possible unsatisfied constraints, whether the unsatisfaction is severe or just medium. The utilitarian SWO is quite satisfying for the use but a powered utilitarism with a high power (e.g. 5, Table 1) is much better as it penalizes the "medium" or "diffuse medium" distributions that have many medium satisfactions (i.e. that require heavy post-editing).

The final use case is the evaluation of a map compared to the previous state of the map. The use case requires a SWO that conveys improvements in its preferences: fewer low utilities and more high utilities. Moreover, a CUF is not necessary as distribution are only compared to the previous one, so all leximin-based SWOs, that do not have a CUF, can be used in this use case. No single SWO seems to be sufficient in this use case, so we propose to use a leximin with poverty line SWO to measure the decrease of low utilities and an iso-elastic SWO with a parameter value close to 0 (e.g. $10^{-5}$, see Table 1 ) to measure the increase of high utilities.

In the next section, experimentations validate the choices made for each use case.

\section{Application to CollaGen Model Generalisations}

The CollaGen model is an automatic framework to carry out iterative generalization procedures that use different existing processes on different parts of the map [1] (Fig. 6). For instance, the cities are first generalized by process 1 , then, rural areas by process 2 and finally roads by process 3 . In CollaGen, the three use cases can occur, so it is possible to experiment the social welfare evaluation framework. In the experiment, a sample with 25 constraints is used including constraints on one object (building minimum area, maintain road initial shape, etc.), on two objects (e.g. minimum distance between buildings) and on group of objects (density of building blocks, 
maintain building alignments, etc.). For instance, the 25 constraints are managed by 13895 monitors in the map city of Fig. 6.

Use case 1 is illustrated by the final output of a 1:50k map, generalized with the CollaGen model (Fig. 7). As the results are very good, the distribution is mainly composed of very satisfied constraints and the Owa welfare SWO gives a value of 5.23 while the utilitarian SWO gives 5.72. The generalization result is slightly damaged artificially on the medium satisfied constraints, to simulate a less good process: the results confirm that Owa welfare is less sensitive to variations in medium satisfactions as they show a bigger decrease in the utilitarian CUF than in the Owa one. Moreover, another alternative generalization is carried out by damaging a few maximum satisfactions that become minimum satisfactions. The experiment shows that the Owa SWO is more sensitive to that, with a bigger decrease of the CUF (0.9 against 0.4$)$. Therefore, Owa seems to be well adapted to the use case 1 .

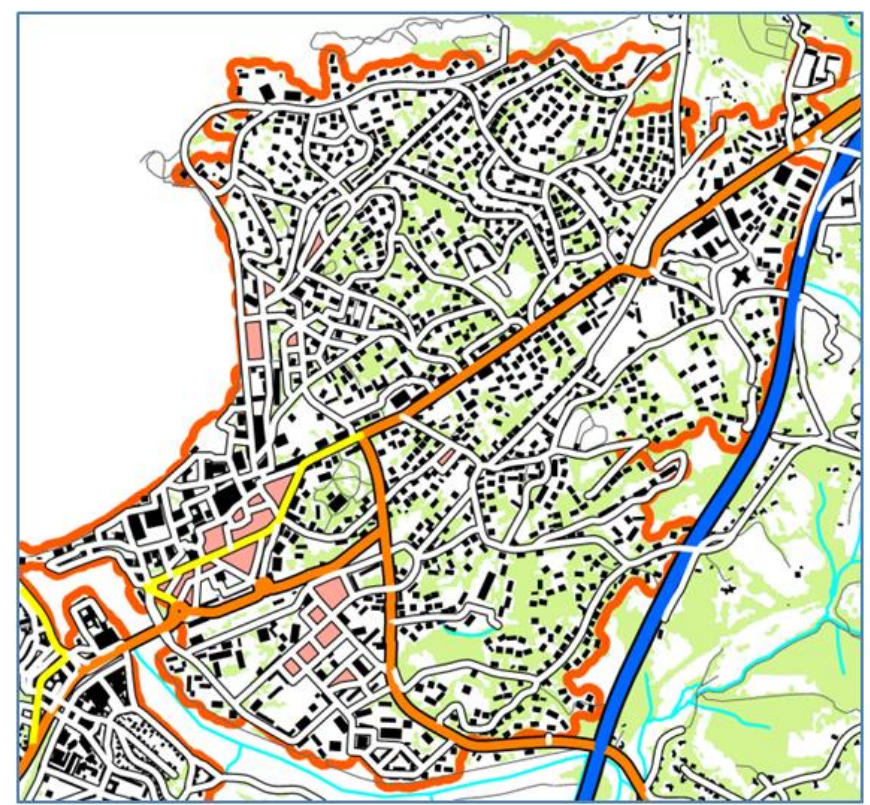

Fig. 6. A generalized city, whose satisfaction distribution contains nearly 14000 monitors.
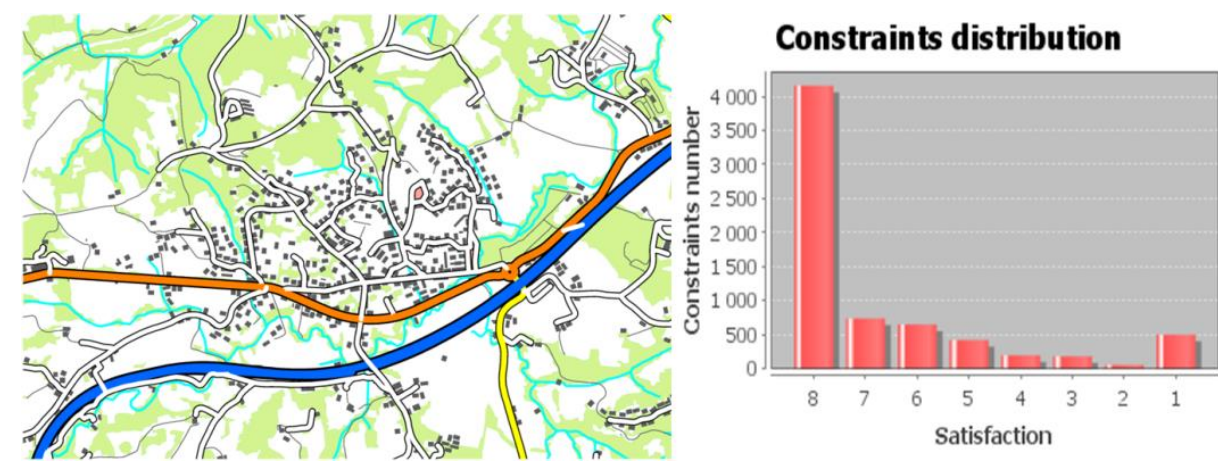

Fig. 7. Use case 1: a very good final output at 1:50k, obtained with CollaGen [1]. This kind of distributions is better comprehended by an Owa SWO.

Use case 2 (final output with manual editing) is illustrated by a rural generalized map by two automatic processes, the second one averaging the constraint satisfaction, which should be penalized by the chosen SWO (Fig. 8). Utilitarian welfare ranks both generalizations the same way (5.70) so it is well adapted to use case 2. On the other hand, powered utilitarian welfare ranks generalization (1) first with a 5.73 CUF against 5.68 for generalization (2), showing its better accommodation to use case 3 . 


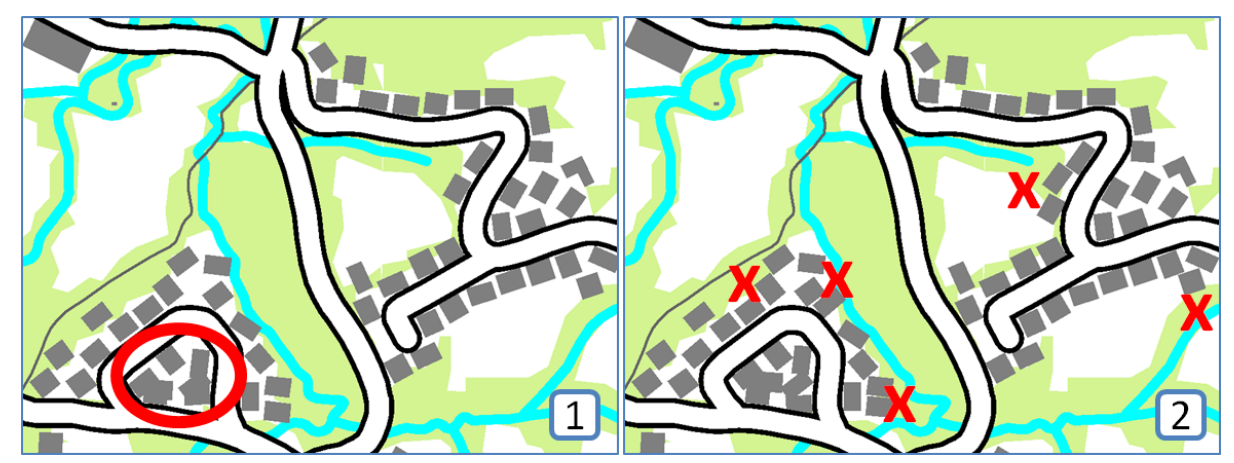

Fig. 8. (1) good for manual editing with few (but major) conflicts in the circled area (2) optimizing generalization, not suited for manual editing (crosses show required minor edits).

Use case 3 is illustrated by the generalization of a city with several iterative processes. The first process generalizes most of the city, then, two alternative processes are used and evaluated with the previous state to assess a significant improvement (Fig. 9). The experiments showed that the leximin with poverty line SWO chosen to assess decreases in low satisfactions is not enough as all generalization results, and even the good ones, remain partly unsatisfied, which limits the differentiations. So, the SWO was adapted to use the quantity of constraints under the poverty line to compare the distributions. Such a change produces good result: while the utilitarian SWO ranks the improvement exactly the same (5.6 against 5.0 for the previous distribution), the adapted leximin SWO considers (D3) as a significant improvement (6.9\% decrease in unsatisfied constraints) and (D2) as negligible ( $2.4 \%$ decrease). The difference is weakened considering the high satisfactions, which is well translated by the Iso SWO that considers the improvement as a bit better in (D3) as in (D2).

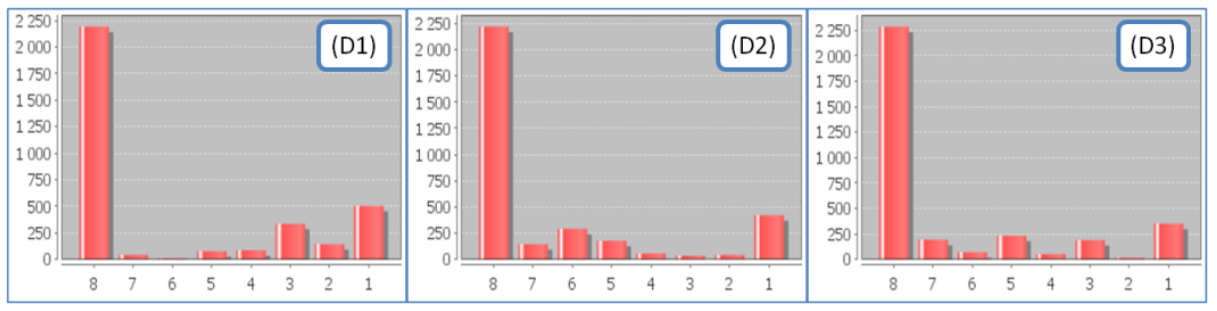

Fig. 9. (1) Distribution after first process. (2) Distribution after alternative process 1: negligible improvement. (3) Distribution after alternative process 2 : it is a significant improvement.

Do the previous applications prove that collective welfare theories significantly improve generalization global evaluation? Several points can be discussed. First, the application of different SWOs to the three use cases shows the advantage of such a vast theory, i.e. its flexibility: we found at least one SWO that fits better than mean, with the purposes of each use case. Moreover, an alternative would be to use multiple criteria decision techniques [19], each constraint being a criterion. But the large number of monitors in a map (Fig. 6) better fits the number of individuals in a society, as multiple criteria decision uses fewer criteria (5 to 15 in most techniques). Another advantage of collective welfare analogy is its expressiveness. For instance, the use of a poverty line in use case 3 is quite expressive. Finally, although differences are not huge, the applications show the sensitivity allowed by collective welfare: it is possible to deal with use cases where mean is not able to give completely satisfying result.

However, there are a lot of SWOs to discriminate. So, it requires intensive testing (e.g. with toy distributions) to find the best SWO for a given use case.

\section{Conclusion and Further Work}

To conclude, we proposed a framework to assess the global legibility of a generalized map, based on a parallel with collective welfare theories. Individual constraints that monitor legibility are considered as individuals in a collective society with a level of satisfaction. In the economical field, several methods (Social Welfare Orderings) exist to measure the global welfare of the society from individual welfare. Several SWOs have been compared in relation to the global evaluation of generalized maps and some have been chosen to meet some use cases demands. The experiments, carried out on generalized topographic maps, validate the use of collective welfare methods. 
To go further, other generalization use cases could be tested, where other SWOs would provide better results than the mean. Moreover the SWOs could be used to assess, on the same data, automatically and manually generalized maps, used as references. It would allow assessing if constraints really convey all specifications, without the softening effect of the mean. Then, the chosen SWOs could be tuned to be even more adapted to the use cases: machine learning on result samples could help to weight the SWOs like in [20]. Finally, it would be interesting to use SWOs to solve the legibility problem of geoportals, studied in [21].

\section{References}

1. Touya, G., Duchêne, C.: CollaGen: Collaboration between automatic cartographic generalisation processes. In: Ruas, A. (ed.) Advances in Cartography and GIScience, pp. 541-558. Springer, Berlin (2011)

2. Mackaness, W. A., Ruas, A.: Evaluation in the map generalisation process. In: Mackaness, W. A., Ruas, A., Sarjakoski, L. T. (eds.) Generalisation of Geographic Information, pp. 89-111. Elsevier, London (2007)

3. Bard, S.: Quality assessment of cartographic generalisation. Transactions in GIS 8 (1), 63-81 (2004)

4. Stoter, J., Burghardt, D., Duchêne, C., Baella, B., Bakker, N., Blok, C., Pla, M., Regnauld, N., Touya, G., Schmid, S.: Methodology for evaluating automated map generalization in commercial software. Computers, Environment and Urban Systems 33 (5), 311-324 (2009)

5. Moulin, H.: Fair Division and Collective Welfare. The MIT Press (2004)

6. Goodchild, M. F., Jeansoulin, R. (eds.): Data Quality in Geographic Information: From Errors to Uncertainty. Hermes, Paris (1998)

7. Ruas, A.: The roles of meso objects for generalisation. In: Proceedings of 9th International Symposium on Spatial Data Handling, vol. 3b, Beijing, China, pp. 50-63 (2000)

8. Ruas, A.: Automatic generalisation project: Learning process from interactive generalisation. OEEPE Official Publication 39 (2001)

9. Zhang, X., Stoter, J., Ai, T., Kraak, M.-J.: Formalization and data enrichment for automated evaluation of building pattern preservation. In: Guilbert, E., Lees, B., Leung, Y. (eds.) Proceedings of Joint International Conference on Theory, Data Handling and Modelling in GeoSpatial Information Science, ISPRS archives, vol. XXXVIII (2010)

10. Likert, R.: A technique for the measurement of attitudes. Archives of Psychology 22 (140), 1-55 (1932)

11. Beard, K.: Constraints on rule formation. In: Buttenfield, B., McMaster, R. (eds.) Map Generalization, pp. 121-135. Longman Pages (1991)

12. Mustière, S., Zucker, J.-D., Saitta, L.: Abstraction-Based machine learning approach to cartographic generalisation. In: Proceedings of 9th International Symposium on Spatial Data Handling, vol. 1a, Beijing, China, pp. 50-63 (2000)

13. Bentham, J.: An Introduction to the Principles of Morals and Legislation. Clarendon Press, Oxford (1789)

14. Nongaillard, A., Mathieu, P., Jaumard, B.: A realistic approach to solve the nash welfare. In: 7th conference on practical applications of agents and Multi-Agent systems. Advances in Intelligent and Soft Computing, vol. 55, pp. 374-382, Springer, Heidelberg (2009)

15. Rawls, J.: A theory of Justice. Belknap, Cambridge (1971)

16. Yager, R. R.: On ordered weighted averaging aggregation operators in multicriteria decisionmaking. IEEE Trans. Syst. Man Cybern. 18, 183-190 (1988)

17. Sen, A.: Rawls versus bentham: An axiomatic examination of the pure distribution problem. Theory and Decision 4 (3), 301-309 (1974)

18. Tungodden, B.: Egalitarianism: Is leximin the only option? Economics and Philosophy 16 (2), 229-245 (2000)

19. Figueira, J., Greco, S., Ehrogott, M. (eds.): Multiple Criteria Decision Analysis: State of the Art Surveys. Springer (2005).

20. Taillandier, P., Gaffuri, J.: Designing generalisation evaluation function through human-machine dialogue. In: Proceedings of GIScience 2010, Zurich (2010)

21. Stigmar, H., Harrie, L.: Evaluation of analytical measures of map legibility. The Cartographic Journal 48 (1), 41-53 (2011) 